C. Aerts, T.R. Bedding, \& J. Christensen-Dalsgaard, eds.

\title{
$\delta$ Scuti Variables with Periods Longer than 0.25 Days
}

\author{
E. Rodríguez
}

Instituto de Astrofísica de Andalucía, CSIC, P.O. Box 3004, E-18080

Granada, Spain

\author{
M. Breger \\ Institut für Astronomie, Universität Wien, Türkenschanzstr. 17, \\ A-1180, Austria
}

\begin{abstract}
Variables listed as $\delta$ Scuti-type pulsators in the R00 catalogue (Rodríguez et al., 2000) with periods longer than $0 .{ }^{d} 25$ are examined on a star-by-star basis, in order to discriminate their true nature ( $\delta$ Scuti, RR Lyr or $\gamma$ Dor-type). From this study, it seems that three stars (DH Peg, UY Cam and YZ Cap) are RR Lyr pulsators rather than $\delta$ Scuti variables. Moreover, two variables (AD Ari and DE Oct) seem to be $\gamma$ Dor variables with the shortest periods known to date $\left(0 .{ }^{d} 2699\right.$ and $0 .{ }^{d} 2778$, respectively).
\end{abstract}

\section{Discussion}

Only a few variables with periods ranging from $0 .{ }^{d} 25$ to $0 .{ }^{d} 3$ are listed in the R00 catalogue (Rodríguez et al., 2000) on $\delta$ Scuti-type pulsators. The true number may be larger, since a selection effect probably exists for pulsators with such periods because of the period overlap with other types of pulsators. Pulsating variables with periods ranging within this interval are very interesting because in this region the evolved Population I $\delta$ Scuti stars and the Population II RRctype variables can coexist. Since $\delta$ Scuti stars include all stars evolving from the main sequence to the giant region (while inside the instability strip), $\delta$ Scuti stars with periods even longer than $0 .{ }^{d} 3$ are expected.

However, due to the rapid evolution of massive stars evolving towards the giant region, the probability of finding a massive $\delta$ Scuti variable in this region of the H-R diagram is small. Consequently, when a new pulsating variable is discovered with a period longer than $0 .{ }^{d} 25$, it is usually assumed that the star is an RR Lyrae-type star situated on the horizontal branch. Only when other parameters are known, such as metallicity, galactic location or spatial motions, is it possible to distinguish between the two groups.

It is also possible to confuse a $\delta$ Scuti star having a relatively long period with the $\gamma$ Dor gravity-mode pulsators, especially among the cool stars between spectral types F0 and F5. Since $\gamma$ Dor stars are on or near the main sequence and the long-period $\delta$ Scuti stars are evolved, knowledge of the luminosity allows us to distinguish between the two classes. 
The aim of this work is to analyze star by star the variables catalogued in the R00 list with periods longer than $0 .^{d} 25$, in order to assign the appropriate type (and, by inference, the evolutionary status). Table 1 lists, in order of increasing periods, the 14 variables listed in the R00 catalogue with periods between $0 .{ }^{d} 25$ and $0 .{ }^{d} 30$, together with some relevant parameters. In this table, $\mathrm{N}$ is the number of detected frequencies. The absolute magnitude $\mathrm{M}_{v}$ has been calculated by two methods (from the parallax given by $\operatorname{HIPPARCOS}\left(\mathrm{M}_{v}(\pi)\right.$; ESA, 1997) and from Strömgren photometry $\mathrm{M}_{v}(\mathrm{ph})$ ), when available. The calculation of $\mathrm{M}_{v}(\pi)$ is restricted to those stars with $\mathrm{s}_{\pi} \leq 0.25$. The photometric absolute magnitudes $\left(\mathrm{M}_{v}(\mathrm{ph})\right)$ have not been determined for stars too luminous $\left(\delta \mathrm{c}_{1}>0 .^{m} 28\right)$.

In the last column of Table 1, we list the most probable identification of each star on the basis of their pulsational behaviours, luminosities, metallicities and light curves. The results suggest that three stars (DH Peg, UY Cam and YZ Cap) are RR Lyr-type pulsators rather than $\delta$ Scuti variables. Moreover, two variables (AD Ari and DE Oct) seem to be $\gamma$ Dor variables with the shortest periods known to date $\left(0 .{ }^{d} 2699\right.$ and $0 .{ }^{d} 2778$, respectively). Multisite coordinated photometric campaigns (including also spectroscopic observations) are presently being carried out for the two latter variables. In the remaining cases, it seems they are $\delta$ Scuti-type variables.

Table 1. Variables in the R00 catalogue with periods $\mathrm{P}>0{ }^{d} 25$

\begin{tabular}{lcccccccc}
\hline \multicolumn{1}{c}{ Star } & $\begin{array}{c}\mathrm{P} \\
(\mathrm{d})\end{array}$ & $\begin{array}{c}\mathrm{V} \\
(\mathrm{mag})\end{array}$ & $\begin{array}{c}\Delta \mathrm{mag}) \\
(\mathrm{mag}\end{array}$ & $\begin{array}{c}\mathrm{M}_{v}(\pi) \\
(\mathrm{mag})\end{array}$ & $\begin{array}{c}\mathrm{M}_{v}(\mathrm{ph}) \\
(\mathrm{mag})\end{array}$ & {$[\mathrm{Me} / \mathrm{H}]$} & Type \\
\hline HN CMa & 0.2501 & 6.59 & 0.01 & 4 & $-0.42( \pm 0.36)$ & & -0.39 & $\delta$ Scuti \\
4996-V5 & 0.251 & 15.0 & 0.36 & 1 & & & & $\delta$ Scuti? \\
DE Lac & 0.2537 & 10.28 & 0.32 & 1 & & 1.18 & 0.23 & $\delta$ Scuti? \\
II-52 & 0.2551 & 16.77 & 0.05 & 1 & & & & $\delta$ Scuti? \\
DH Peg & 0.2555 & 9.56 & 0.50 & 1 & & & -0.80 & RR Lyrae \\
UY Cam & 0.2670 & 11.44 & 0.34 & 1 & & & -0.72 & RR Lyrae \\
V1719 Cyg & 0.2673 & 8.01 & 0.31 & 2 & $1.05( \pm 0.36)$ & 0.73 & 0.25 & $\delta$ Scuti \\
AD Ari & 0.2699 & 7.43 & 0.06 & 1 & $2.79( \pm 0.16)$ & 2.44 & -0.06 & $\gamma$ Dor? \\
V388 Cep & 0.2717 & 5.56 & 0.04 & 5 & $0.37( \pm 0.14)$ & & -0.08 & $\delta$ Scuti \\
S Eri & 0.273 & 4.78 & 0.03 & 1 & $0.03( \pm 0.14)$ & & -0.07 & $\delta$ Scuti \\
YZ Cap & 0.2735 & 11.38 & 0.49 & 1 & & & & RR Lyrae \\
DE Oct & 0.2778 & 9.15 & 0.07 & 1 & $2.2( \pm 0.5)$ & 2.50 & 0.00 & $\gamma$ Dor? \\
7654-501 & 0.278 & 14.42 & 0.02 & 1 & & & & $\delta$ Scuti \\
SS Psc & 0.2878 & 10.99 & 0.39 & 1 & & & 0.03 & $\delta$ Scuti? \\
\hline
\end{tabular}

\section{References}

ESA 1997, The Hipparcos and Tycho Catalogues, ESA SP-1200

Rodríguez, E., López-González, M. J., \& López de Coca, P. 2000, A\&AS, 144, 469 